\title{
LXVII. Selective reflexion, scattering and absorption by resonating gas molecules
}

\section{R.W. Wood}

To cite this article: R.W. Wood (1912) LXVII. Selective reflexion, scattering and absorption by resonating gas molecules, Philosophical Magazine Series 6, 23:137, 689-714, DOI: 10.1080/14786440508637267

To link to this article: http://dx.doi.org/10.1080/14786440508637267

册 Published online: 08 Jun 2010.

Submit your article to this journal $\pi$

山 Article views: 10

Q View related articles $\square$

Citing articles: 25 View citing articles $\sqsubset$ 
LONDON, EDINBURGH, AND DUBLIN

\title{
PHILOSOPHICAL MAGAZINE
}

\author{
AND \\ JOURNAL OF SCIENCE.
}

[SIXTH SERIES.]

$M A Y 191 \%$.

I.XVII. Selective Reflexion, Stattering and Absorption by Resonating Gas Molecules. By R. W. Wood, Professor of Experimental Physics, Jolens Hopkins University, and Adams Research Fellow of Columbia University *.

[Plates X.-XII.]

T $\mathrm{N}$ the present paper I shall deal with phenomena which, 1 up to the present time, have never been made the subject of experimental investigation; and though elaborate mathematical treatments have been given by Planck, Lord Rayleigh, Schuster, Lamb, and others, no experimental data have ever been obtained. Through the discovery that the vapour of mercury, at room temperature, emits a brilliant resonance radiation when stimulated by monochromatic ultraviolet light of wave-length corresponding exactly to that of its so-called absorption line $(\lambda=2536)$, I have been enabled at last to obtain quantitative data on the subject.

It has been found that when the vapour is in a high vacuum there is no true absorption, the energy diverted from the primary beam of light being wholly scattered, as imagined by Planck in his treatment of the theory of absorption. The presence of a small quantity of air or other gas has been found to introduce the factor of true ahsorption, or the conversion of the energy of the light-waves into heat, and the ratio of the amount of energy scattered to that absorbed as a function of the pressure of the gas with which

- Communicated by the Author.

Pkil. Mag. S. 6. Vol. 23. No. 13T. May 1912. 
the mercury vapour is mixed has been determined. A very remarkable secondary resonance radiation of the gas not directly illuminated by the primary beam, but stimulated by the light which comes from the directly exciter molecules, has been discovered, the study of which has enabled me to draw the conclusion that there is no true absorption except when the pressure is raised by the presence of air.

I have also succeeded in passing by gradual stages from the condition in which the resonance radiation is diffusely scattered to that in which, owing to the proximity of the molecules, it is regularly reflected from the surface of the gas mass. A resonance lamp has been constructed which emits light probably more homogeneous than that obtained from any source hitherto known, by the light of which I have succeeded in photographing the vapour rising from a drop of warm mercury into the air of the room like black smoke. So sensitive is the light of this lamp to the presence of mercury vapour, that I have been obliged to ventilate my room before each experiment, owing to the presence of mercury in the cracks on the floor, for the light of the lamp is reduced to one-half of its intensity by traversing a layer of mercury vapour at room temperature only $5 \mathrm{~mm}$. in thickness.

The following topics will be disctissed :-

Introduction.

Resonance radiation of mercury vapour at room temperature.

Energy diverted from the primary beam.

Primary and Secondary Resonanee Radiation.

Destruction of the Secondary Radiation by molecnlar collisions.

Probability that the molecules continue to emit light after they leave the region traversed by the primary beam.

Ratio between Scattered and Absorbed Light as a function of the pressure of the gas.

Transition from Diffuse Scattering to Regular Reflexion.

Absence of all traces of Polarization.

Experiments with the Resonance Lamp.

\section{Introduction.}

The scattering of light by gas molecnles has been the subject of mathematical treatments, but practically no experimental work has been done upon the subject, and we have no data whaterer regarding the actual amount of energy 
diverted from the primary beam by a molecule in exact resonance with the light-waves which excite it.

Lord Rayleigh has interested himself chiefly with the scattering which may occur when the molecules are not in resonance, as is the case in the scattering of the blue light by the molecules of the atmosphere. This case has been experimentally investigated by Abbott, who has measured the atmospheric absorption from the summit of Mount Wilson. Of greater interest, however, is the scattering of molecules resonating under the influence of the light-waves. But few cases of this are known, though this action has been made the basis of the theory of absorption developed by Planck. Some years ago I found that the non-luminous vapour of sodium at a comparatively low temperature, when illuminated by the light from a powerful sodiam flame, re-emitted light of the same wave-length in all directions. 'This re-emission of light, without change of wave-length, by molecules of an absorbing gas, I named resonance radiation, to distinguish it from fluorescence, where there is in general an increase in the wave-length. Sodium vapour is, however, almost impossible to handle in a satisfactory manner, and is in consequence unsuitable for a quantitative investigation of the subject.

About two years ago I discovered that mercury vapour acts in the same manner, except that the phenomenon occurs in the ultra-violet region at the absorption line 2536 . I first observed the resonance radiation of this rapour at room temperature in an exhausted silica bulb, the pressure of the mercury vapour being $0.001 \mathrm{~mm}$.

This vapour appeared to be the ideal medium for a careful study of the subject in all of its aspects, except for the fact that everything has to be done by photography. The work has turned out even better than I had hoped, and I have been able to pass gradually from the case where the scattered light is radiated in all directions by the highly rarefied gas to that in which it is regularly reflected from the surface of the gas under high pressure.

I have already described the selective reflexion of monochromatic light by mercury vapour (this journal, vol. xviii. p. 187,1909 ), in a paper which I shall have occasion to refer to later on.

Although I first observed the resonance radiation of mercury vapour nearly two years ago, I published nothing on the subject, as no quantitative data had been obtained. The observation was made in the following way. A small drop of mercury was introduced into a tube of fused quartz, 


\section{Prof. R. W. Wood on Selective Reflewion, Secuttering}

closed by end plates of the same substance which had been ground flat and polished. These plates were fused to the ends of the tube, which had been flared out in order to prevent spoiling the figure of the central portion of the plates by fusion. The tube is shown in Pl. X. fig. 1, and was designed for a study of the dispersion of the vapour by an interferometer method, an investigation of which will shortly be commenced. It was made by Heraeus and turned out quite as well as I had hoped, for I have had no difficulty in forming interference fringes with one beam of liglit traversing the tube, showing that the process of fusing on the end plates did not damage their optical quality. The tube was highly exhausted and sealed, and the light of a quartz mercury arc focussed along the axis of the tube. The tube was now photographed from the side with a camera furnished with a lens of quartz, which was constructed in a few minutes from an old box used for storing photographic negatives. A quartz lens of about $18 \mathrm{~cm}$. focus was fitted into a hole nuade in the bottom of the box, which was used standing on its side. A strip of thick sheet brass was cut to the same width as the plates for which the box was made. To this strip was soldered another strip in which a circular aperture $8 \mathrm{~cm}$. in diameter had been cut. This strip stood vertically in the box, and was provided with two spring clips, or supporting clamis, to hold the plate in place against the circular aperture. The camera was focussed by sliding the strip along in the grooves which supported it. (These grooves were made originally for holding the plates.) The correct focus for the invisible rays with which we are concerned in the present paper was found by pointing the camera at the slit which had been mounted in the focal plane of the quartz spectrograph (to convert it into a monochromator), and observing the focus with a piece of uranium glass. The distance from the lens to the slit from which the ultra-violet rays diverged was then measured. This gives the correct distance at which the object to be photographed must be placed in order to be properly focussed. The eamera is shown in fig. 1 a. I have found that much time is saved by the use of a camera of this description, for the plates can be cut carelessly and in a hurry, as they do not have to be of an exact size, and no time is lost in loading or unloading a plate-holder. In ultra-vio'et work of this description, where the sonrces of error and all other unexpected troubles and difficulties have to be located by photography, the time saved in this way amounts to a good deal. In the present work I have taken about six bundred 
photographs, and have saved the many hours that would have been spent in fussing with plate-holders and miss-fit plates. The hinged back of the box can be swung aside and the plate inserted in the supporting clamps in a few seconds, the room being darkened of course.

Fig. I $a$.
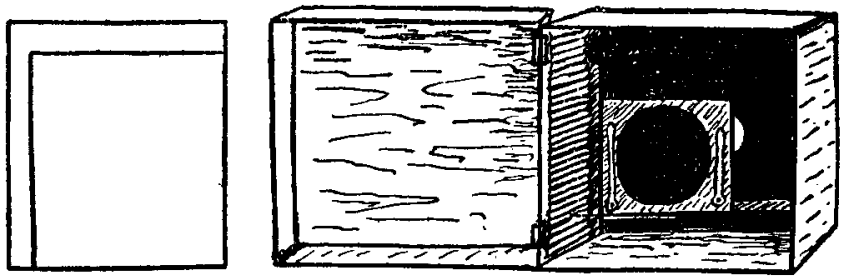

The Westinghouse quartz arc was enclosed in a box and the collimator of the quartz spectrograph introduced through a small hole. In this way diffused light, which would have spoiled everything, was shut out. The lamp was started by pulling a string which passed through a small hole in the top of the box, and extinguished at the right moment by opening a switch.

The monochromatic light from the slit of the quarts spectrograph was either made parallel, or brought to a focus by means of a pair of quartz lenses of $30 \mathrm{~cm}$. focus each, the condition of the pencil of rays being tested with the uranium glass plate.

The photograph taken of the long tube closed with flat quartz plates showed an image of the cone of rays traversing the high vacuum precisely as if the tube were filled with dense smoke. 'The tube was at room temperature, and the density of the mercury vapour was about $0.001 \mathrm{~mm}$., nevertheless an exposure of fifteen or twenty seconds was all that was necessary.

Owing to my absence from Baltimore $I$ was unable to take up the work again until this winter. In the meantime I had secured a Westinghouse Cooper-Hewitt quartz mercury arc which was very much more powerful than my old Heraeus lamp. These quartz arcs emit the strong line of wave-length 2536 , and it is this light alone which excites the resonance radiation.

To my surprise, on repeating the experiment of two years ago with the new arc lamp, I found no trace whatever of the luminous cone of light, even with an exposure of several minutes. On looking up my notes, however, I found that in 


\section{Prof. R. W. Wood on Selective Reflexion, Scattering}

the earlier experiments $J$ excited the lamp with a high potential current from a transformer, running it at a comparatively low temperature. It immediately occurred to me that in the present ease I was working with the lamp at high temperature, and that the 2536 line was very likely reversed, the wave-length necessary for the excitation of the resonance radiation being removed by absorption. I according!y allowed the lamp to become quite cold, and made my exposure when the lamp was first ligbted, when its light is quite violet in colour. On developing the plate 1 found that a five seconds exposure gave me a more intense cone of light than anything that $I$ had ever observed betore. It was absolutely black on the negative. Moreover, the vapour outside of the cone of focussed rays appeared to be glowing at the end of the tube where the beam passed in. As the fused quartz is somewhat fluorescent under the action of the rays emitted by the lamp, it was necessary to prove that this light did not come from the walls of the tube. That such is not the case I shall show when we come to subsequent experiments.

A photograph of the resonance radiation in the tube is reproduced on $\mathrm{PI}$. XI. fig. 1 .

It was next necessary to observe the spectrum of the light emitted by the mercury vapour, and the slit of a quartz spectrograph was opened wide and brought up close to the silica tube. Two photographs were made-the first of ten seconds exposure immediately on starting the Westinghouse lamp; the second, also of ten seconds after the lamp had been running half a minute. These photographs are shown in PI. XI. fig. 2. In the second spectrum the broadened image of the slit is shown at the 2536 line (indicated), the other lines appearing narrow. These lines result from the circumstance that some direct light from the arc illuminated one of the jaws of the slit. The upper spectrum, taken after the lamp had been in action for half a minute, is identical with the second, except that the broad image of the slit is absent, showing that the resonance radiation has disappeared. I then made a series of four spectra taken one after the other. 'These showed that the resonance radiation was very strong during the first five seconds of the lamp's operation, quite faint during the second five seconds operation, the merest trace during the third five seconds, and completely gone in the next five seconds. In all of the subsequent work I accordingly allowed the lamp to become quite cold, and extinguished it after a run of exactly five seconds. This insured uniformity in the exposures. The slit of the spectro- 
graph was now made as narrow as possible and five exposures were superposed of five seconds each, the direct light from the arc being screened from the slit. The spectrum showed only the 2536 line, exceedingly narrow and sharp, thus proving that we are dealing with a very beautiful case of pure resonance radiation. In fact, I suspect that this radi. ation will be found to be the most homogeneous which we have, for the vapour is not only at a lower pressure than is usual in vacuum-tuhes, but it is at room temperature. In Pl. XI. fig. 2, the third spectrum is that of the resonating vapour, the fourth spectrum that of the mercury arc.

I next investigated the effect of raising the temperature of the tube which was mounted in a small air-bath. It was found, as was to be expected, that, as the temperature rose, the cone of emitted light became shorter and brighter, until it finally disappeared, the emitted light coming from the inner surface of the plate where the incident beam of light entered. As no especial significance is attached to the records obtained in this way, I shall postpone any further discussion of the effects of increasing the density of the vapour until I take up the subjoct of the transition from diffuse scattering of the emitted light to its recombination into a regularly reflected wave, which can be accomplished by increasing sufficiently the density of the vapour.

We will now take up the important subject of the amount of energy diverted from the incident beam by the resonating gas molecules.

\section{Amount of Energy diverted from the Primary Beam.}

It is clear at the outset that if we wish to determine the amount of energy diverted by the resonators when they are in exact synchronism with the light-waves, it is useless to make observations upon the intensity of the light after it has suffered transmission through the vapour, even if we are dealing with what we are accustomed to call monochromatic ljght. All spectrum lines have a finite width, and the particular frequency scattered by the resonating molecules may constitute but a small fraction of the total energy of the spectrum line used to excite the vaponr; in other words, it is only the centre of the line that is effective in exciting resonance, the edges of the line not being reduced in intensity by the transmission through the gas. What we wish to determine is the reduction in intensity of that portion of the line, or in other words the frequency, which is capable of exciting the natural period of vibration of the 


\section{Prof. R. W. Wood on Selective Reftexion, Scattering}

molecule. It appeared to $\mathrm{mg}$ that the most direct way of investigating this question was to take the intensity of the cone of light as the measure of the intensity of the primaly beam, for there appears to be no doubt but that the intensity of the resonance radiation is proportional to the intensity of that particular frequency in the exciting light which is capable of setting up resonance. I accordingly made a very large number of photographs with different times of exposure and different vapour densities, and measured the photographic density of the inage at different distances from the point where the light entered the vapour. This gives us a measure of the rate at which the vapour cuts down the amplitude of the exciting frequency as the wave moves through the medium. The method by which these measurements were made will be described presently, and for the present 1 need only point out that the usual precautions necessary in photometric work by photography were taken. The mercury vapour in this case was not contained in the quartz tube, for it was impossible to get a good image of the excited region close up to the point at which the beam entered on account of the irregular refraction by the wall of the tube. A rectangular box of brass with windows of crystal quartz plates, which I shall describe presently, was used, and by paying attention to adjustments, it was possible to get beautifully sharp image of the excited region close up to the surface of the window through which the light entered. I made measurements on fully a dozen plates taken on different days, and found that the intensity of the primary beam was reduced to one-half of its value after travelling for a distance of $5 \mathrm{~mm}$. in mercury vapour at a pressure of $0.001 \mathrm{~mm}$. The smallest value found was $4 \mathrm{~mm}$. and the largest $6 \mathrm{~mm}$. for a reduction to half intensity, a variation due without donbt to the circumstance that the room temperature varied by several degrees from day to day. This variation in the temperature of the room was just about sufficient to account for the slight differences observed, and we can safely assign the value of $5 \mathrm{~mm}$. for a temperature of $22^{\circ} \mathrm{C}$. Measuring the intensity along the path of the beam it was found that the ordinary litw of absorption was very closely followed, $i . e$. a $10 \mathrm{~mm}$. layer reduced the intensity to $1 / 4$, and a $15 \mathrm{~mm}$. one reduced it to $1 / 8$ of its original value. It appeared to me, however, that the reduction of the intensity did not follow this law exactly, but that the intensity was a trifle greater after $15 \mathrm{~mm}$. had been traversed than the intensity calculated on the assumption that every 
$5 \mathrm{~mm}$. effected a reduction of $1 / 2$. This is in agreement with a calculition made by Schuster in his paper on "Radiation through a Foggy Atmosphere" (Astrophysical Journ. vol. xxi.p. 6,1905 ), though the differences in this case are not much larger than the probable errors in the measurements. The beain of light which entered the cell was made accurately parallel by means of a quartz lens and passed through a square aperture (measuring $5 \mathrm{~mm}$. on a side) perforated in a black card. In this way a beam of uniform cross-section was obtained, which was of course necessary if the measurements were to be of any value. Only the ultraviolet 2536 light entered the cell, a quartz spectrograph being used as a monochromator.

We are now in a position to consider the amount of energy diverted from the primary beam by each molecule.

Lamb, in his theoretical treatment of the absorption of light by a gas, published in the Stokes Commemoration of the Camb. Phil. Soc., sums up a calculation in the following words :- " Hence in the case of exact synchronism, each molecule of gas would, if it acted independently, divert per unit of time nearly half as much energy as in the primary waves crosses a square whose side is equal to the wavelength." This means, if I am not mistaken, that if we had a density such that there was one molecule in each cube the sides of which were equal to the wave-length, the intensity of the light would be reduced by $1 / 2$ by traversing a single layer of molecules, while a density ten or twenty times as great as this ought to give selective reflexion, since the wave would be practically stopped before penetrating to a depth of more than a small fraction of a wave-length.

Let us now compare this calculation with the values which have been determined. At a pressure of $0.001 \mathrm{~mm}$., which is about the pressure used, the average molecular distance is such that we shall have on the average one molecula of mercury in every cube the sides of which are only very little larger than the wave-length (or more exactly $0.0003 \mathrm{~mm}$.), which quantity divided into $5 \mathrm{mms}$., the distance traversed for a reduction of intensity equal to $1 / 2$, gives us 16,000 , that is to say 16,000 molecules must be passed before one half of the energy is removed from a square element on the wave-front measuring $\lambda$ on each side.

Of course this calculation is made on the assumption that all of the molecules are equally effective in scattering the light. It is however possible, eren probable, that but a small percentage are, at any giren moment, in the condition 
to act as resonators. Experiments on the dispersion and magnetic rotation of metallic vapours and luminescent hydrogen give evidence that but a small percentage of the molecules are at any instant concerned in the production of the phenomena in question.

\section{Primary and Secondary Resonance Radiation.}

Photographs of the luminous cone of inercury vapour at room temperature contained in the quartz tube appeared to prove that the vapour outside of the cone of vapour directly excited by the primary beam was itself luminous. It was observed, however, that the fused quartz phosphoresces with a violet light under the influence of the ultra-violet light, and I did not feel perfectly sure that the light did not come from the wall of the tube. To eliminate such a possibility a hollow box of brass was constructed (Pl. X. fig. 2), two adjacent sides of which were left open, and closed with thin plates of quartz (crystal) which is not phosphorescent. The inside of the box was heavily smoked, and the plates cemented in place with sealing-wax. A drop of mercury was introduced and the interior of the box put in communication with a Gaede pump and exhausted. The ultra-violet light was focussed at the centre of the box, entering through one of the quartz plates, and the resonance radiation photographed from the side through the other plate. It was found that, unless the pressure of the air was less than three or four millimetres, no trace of any secondary radiation was present. On lowering the pressure of the residual air it developed rapidly however, and after the pump had been in operation for several uinutes, the luminous glow filled the entire interior of the box, the luminous cone being nearly lost on the strongly luminous background. With correctly timed exposures the directly excited resonance radiation is always four or five times brighter than the secondary radiation. Over exposure may, however, increase the effect of the secondary until it equals that of the primary, causing the outlines of the primary beam to disappear almust completely, as in the first picture in fig. $4, \mathrm{Pl}$. Xl.

The intensity of the secondary radiation depends upon the cross-section of the primary beam, as does also the rate at which its intensity diminishes with increasing distance from the primary rays. With an exciting beam of square crosssection $\left(5 \mathrm{~mm} .^{2}\right)$ the intensity of the secondary radiation half a millimetre from the edge of the beam was found to be 
nearly $\frac{1}{3}$ of the intensity of the adjacent primary radiation. Its intensity fell off with increasing distance as follows :-

$\begin{array}{ll}\text { Distance. } & \text { Ratio. } \\ 0.5 \mathrm{~mm} . & 1 / 3 \\ 1.5, " & 1 / 6 \\ 2.5 \quad " & 1 / 10 \\ 3.5, & 1 / 30\end{array}$

Four photographs of the phenomenon are reproduced on Pl. XI. fig. 4. A vacuum-tube was put in circuit with the tube leading to the pump, to serve as an auxiliary manometer, and it was found that the secondary radiation did not appear in its maximum intensity until the green fluorescence due to cathode rays appeared in the vacuum-tube.

The arrangement of the apparatus is shown in Pl. X. fig. 2, which requires no explanation. It is clear. from the photographs that when the mercury vapour is in the highest possible vacuum, the light which it emits is capable of exciting a secondary radiation in the surrounding vapour which lies wholly outside of the path of the primary exciting beam. The presence of air at 4 or $5 \mathrm{~mm}$. pressure, while it materially decreases the intensity of the primary resonance radiation, causes the secondary radiation to disappear entirely. In the four photographs which are reproduced I bave recorded the pressure of the residual air in each case. To make sure that the disappearance of the secondary radiation was not due to a weakening of the primary radiation, I gave an exposure of four times the nomal one, with air at $4 \mathrm{~mm}$., and though the cone was very much blacker on the plate than on any of the others, there was no trace of any luminosity in the surrounding vapour.

This action of a small trace of air is most remarkable, and it is of the utmost importance to determine the explanation. Although the vapour which is in the path of the primary beam glows with diminished brilliancy, the light which it gives out seems powerless to excite the surrounding vapour to luminosity. It seemed possible that the damping due to molecular collisions rendered the emitted light less homogeneous and therefore incapable of exciting the vapour, in spite of the fact that the alsorption band must be regarded as broadened to a similar amount. This point is one which requires very careful investigation, and if the cause can be found much light may be thrown upon the nature of the effects of molecalar collisions upon radiation. It occurred 


\section{Prof. R. W. Wood on Selective Reflexion, Scattering}

to me that it might be possible that the emission of light by the vapour surrounding the primary cone might not be due to light emitted by the vapour directly excited, but to the circumstance that, owing to the great length of the mean free path, the mercury molecules might continue to ernit light after flying outside of the limits of the primary beam. 'The action of a small amount of air in destroying the luminosity would follow at once as a result of the reduction in the length of the free path, the luminosity being destroyed by the collisions.

\section{Probability that the Molecules continue to emit Light after they leave the region traversed by the Primary Beam.}

Fortunately this is an hypothesis which is very easily tested by experiment, for we have only to divide the cell into two compartments by means of a partition of quartz, passing the exciting beam immediately below and close to the under side of the horizontal purtition. The partition will stop the moving molecules, but will transmit the light emitted by the vapour illuminated by the primary bearn. A small plate of quartz $1.5 \mathrm{~mm}$. in thickness was cemented to the front window of the cell as shown in Pl. X. fig. 3, and the cell exhausted until nothing but the green phosphorescence was visible in the discharge-tube. The resulting photograph showed that there was considerable luminosity in the upper chamber, but it appeared to be of somewhat less intensity than that of the vapour in the lower chamber. The photograph, however, was not very satisfactory owing to the thickness of the partition. The cell was accordingly made over again, with a partition consisting of a quartz plate only $\frac{1}{4} \mathrm{~mm}$. in thickness, and the same phenomenon was found, somewhat greater intensity of the secondary radiation in the compartment traversed by the exciting beam. Calculation of the reflecting power of the quartz plate from its refractive index for the wave-length in question showed that a reduction of intensity of 10 per cent. was to be expected from this circumstance, but this did not seem to be sufficient to explain the observed diminution of the intensity; and careful measurements of the intensity of the secondary radiation at equal distances from the centre of the primary beam were made by comparison with a plate exposed in strips by gradually increasing amounts of time, which was developed with a photograph of the cell for exactly the same length of time. These measurements showed that the effect of the thin partition was to reduce the 
intensity of the secondary radiation by an amount varying from 25 to 30 per cent. This appears to be very strong evidence of the persistence of the lumirosity of the molecule after it leaves the region traversed by the exciting beam. Direct evidence of the phenomenon is much to be desired, but I have been unable to think of any method, for we cannot make a screen which will transmit the moving molectles but cut off the light.

It is evident, however, that the primary cause of the destruction of the intensity of the secondary radiation by the presence of air at three or four millimetres pressure is to be songht elsewhere, as fully 75 per cent. of the secondary radiation results from the light of the primary. I shall now show that the effect of the air in reducing the intensity of the secondary radiation results from the introduction of the factor of true absorption as contrasted with molecular scattering.

\section{Molecular Scattering and True Absorption. Katio of the two quantities.}

It is easy to see that, if true absorption occurs as well as scattering, the intensity of the secondary radiation will be greatly diminished in intensity. When the vapour is in at vacuum of less than $.01 \mathrm{~mm}$. it is probable that the energy diverted from the primary exciting beam is all scattered, and no true absorption occurs. We should of course find what appeared to be an absorption line in the spectrum of the transmitted light, and yet the molecules would not be absorbing energy but merely diverting it from the primary beam and sending it out in all directions. The molecules lying in the path of the beam will glow with a certain intensity, while those which lie outside of the path of the beam will be illuminated by the radiating molecules which are directly excited, and will in consequence emit a light of a lesser intensity. Suppose now that by the introduction of air at a pressure of $5 \mathrm{~mm}$. the intensity of the light emitted by the directly excited molecules is ieduced to $1 / 3$ of its original value. By means of a threefold increase in the intensity of the exciting light we can raise this intensity to its original value, so that the same amount of light is available for the excitation of the secondary radiation as before. 'The intensity of the secondary radiation excited under these circumstances will, however, be only one-third of its former value, since two-thirds of the energy received from the directly excited molecnles is transformed into heat by the true absorption which has been introduced by the 
presence of the air. The intensity of the secondary resonance radiation in comparison with that of the primary will consequently be much less (one-third) than when the mercury vapour was in a high vacaum. This hypothesis was tested by experiment and practically proven. In the first place a very careful series of measurements was made of the reduction in the intensity of the primary resonance radiation by the introduction of air. The pressure of the air was measured with a McLeod gange, and the duration and intensities of the excitation were made as nearly equal as possible.

The exposures were all made on the same plate, which was pushed along in the supporting clamps, the mercury lamp being allowed to cool down completely between exposures. To insure against accidental errors, a large number of plates were exposed, and the measurements made from each were compared. One of these plates is reproduced on Pl. XI. fig. 12. The air pressure in the cell is marked on each picture. The exciting beam enters the cell from the right, and two thirds of the quartz window was screened off, so that a number of exposures could be made on the same plate. In the first picture (pressure $0.01 \mathrm{~mm}$.) the secondary radiation from the region not excited by the primary beam is very conspicuous, less so in the second and nenrly gone in the third. The intensities of the primary radiation at the point where the incident beam entered the cell was measured by comparing the density of the negative with the density of a plate exposed in strips for times increasing gradually from 5 to 300 , which plate was developed simultaneously with the other. The comparison was made in a very simple way which, with practice, was susceptible of considerable accuracy. A clean cut was made with a sharp knife through the film on each plate, perpendicular to the direction of the strips on the comparison plate, and through the region at which the density was to be measured on the other plate. The film was removed along one side of the cut in each case, exposing the clear glass. The two plates were now placed in contact film to film, and the comparison strip slid along until a perfect density match was made with the photograph, the dividing line between the two disappearing. In practice it was found advantageous to cover the plates with a black card perforated with a small rectangular aperture, which was brought over the region under investigation, and the best match could be made by throwing the eye a trifle out of focus, thus causing the very narrow line separating the two halves of the field to disappear. 
and Absorption by Resonating Gas Molecules.

The values found are given in the following table.

\begin{tabular}{|c|c|c|}
\hline $\begin{array}{c}\text { Air pressure } \\
\text { in mm. }\end{array}$ & $\begin{array}{c}\text { Intensity of primary } \\
\text { resonance radiation. }\end{array}$ & Absorbed energy. \\
\hline 01 & 300 & \\
$\cdot 45$ & 230 & 0 \\
$1 \cdot 10$ & 200 & 70 \\
$2 \cdot 20$ & 170 & 100 \\
$6 \cdot 20$ & 100 & 200 \\
$9 \cdot 50$ & 70 & 230 \\
$14 \cdot 20$ & 50 & 250 \\
$18 \cdot 00$ & 40 & 260 \\
$32 \cdot 00$ & 12 & 288 \\
\hline
\end{tabular}

If we plot these values, taking intensities as ordinates and air pressures as abscissæ, we obtain a curve practically identical with the curve obtained with iodine vapour, which shows that the effect of the air upon the intensity of the emitted radiation is about the same in the two cases. In the third column I have given the amounts of the energy absorbed in each case. These values are mereiy the differences between the amounts of the emitted energies and the energy emitted when the vapour is in a high vacuum (300), and are calculated on the assumption that the total energy diverted from the primary beam is the same in the two cases, i.e., that the presence of the air does not influence the amount of energy removed from the beam by the resonating gas molecules.

That this is in reality the case was shown by the following experiment. A double cell, Pl. X. fig. $4 b$, was made by soldering a cross partition along a diameter of a short section of large brass tube, the ends of which were closed with quartz windows. The length of the tube was $17 \mathrm{~mm}$., and the diameler $30 \mathrm{~mm}$., and two small brass tubes permitted either compartment to be exhausted to any desired pressure. In measuring the energy diverted from the primary beam by the vapour, we must be certain that we use light which is in exact synchronism with the resonating molecules. The light must be far more homogeneous than the ray isolated by the quartz spectrograph from the light of the mercury arc. I used therefore what I shall hereafter refer to as the resonance lamp, a small quartz bulb, closed at the bottom with a flat plate of polished fused quartz, which was fused on in the same manner as the end plates of the long tube previously described. This bulb contained a drop of inercury, and was highly exhausted and sealed. The light from the quartz spectrograph 


\section{Prof. R. W. Wood on Selective Refexion, Scattering}

was focussed through the side of the bulb as close to the rentre of the flat bottom as possible. The adjustments were made by means of a small piece of uranium glass, which enables one to locate the path of the rays by its phosphorescence. It is most important to prevent the light which is reflected from the walls of the bulb from getting at the photographic plate. This gave a good deal of trouble, but by means of the device shown in Pl. X. fig. $4 a$, it was practically eliminated. A wooden box was made, measuring $40 \times 10 \times 10 \mathrm{~cm}$., and a large circular hole ent in one end which was covered with a cone of black cardboard made by cutting out a circular disk, cutting along a radius and pasting the cut edges together, overlapping them about $2 \mathrm{~cm}$. A small round hole was burned through the apex of the cone, and this was placed against the flat bottom of the resonance lamp. A quartz lens mounted in a partition of the box rendered the rays which rame from the resonance lamp through the small hole parallel, so that the intensity of the light after its passage throrgh the cell could be recorded close to the cell or at a distance from it. The importance of doing this is apparent from the following considerations. If we place a photographic plate close against the double cell containing the vapour, it will be illuminated by the primary beam which has traversed the cell and also by the scattered resonance radiation. If, however, we place the plate at a distance, say at the other end of the box, the primary rays, heing parallel, will reach it with undiminished intensity, while the effect of the scattered radiation will be negligible, since its intensity diminishes according to the law of inverse squares. No difference should be found with the plate in the two positions for the light which has gone through the compartment containing air and mercury vapour, since, as we have seen, the presence of the air destroys the resonance radiation. The experiment was made in the following way. One compartment of the double cell was highly exhausted and the other to a pressure of $3 \mathrm{~cm}$. A strip of photographic plate $1.5 \mathrm{~cm}$. in width was mounted close to the cell and received the light which had traversed the lower half of each compartment. A larger plate was mounted at the other end of the box, and received the light which passed ahove the first plate, and had traversed the upper halves of the compartments. Thus four records were obtained at once under precisely similar conditions as regards exposure-time and development. Much trouble was experienced in getting things adjusted so that the intersity close to the lens and at the end of the box came out the same with the cell removed, which is of course a necessary freliminary experiment. It was finally found that the air of 
the room contained enough mercury vapour to reduce the intensity of the light from the resonance lamp by nearly one half as it traversed the length of the box.

This is not so surprising when we remember that the earlier experiments showed that the primary beam was reduced to one half of its intensity (i.e., the frequency capable of exciting resonance was) by traversing $5 \mathrm{~mm}$. of the saturated vapour at room temperature. The trouble was overcome by opening the windows and thoroughly ventilating the room before each experiment. One is reminded of the trouble experienced in carrying on certain investigations in laboratories which have become infected by radium!

One of the photographs obtained with the double cell is reproduced on $\mathrm{Pl}$. XI. fig. 3. In this case one compartment was tree from mercury vapour, while the other contained it. This particular cell was, however, only $8 \mathrm{~mm}$. in thickness, and gives a good idea of the powerful absorption of the highly homogeneous light from the resonance lamp by a shallow layer of the vapour at room temperature. The other photographs showed that the energy diverted from the primary beam was the same for mercury in a high vacuum and in air at $3 \mathrm{~cm}$. pressure, so that the calculation of the absorptionscattering ratio which I gave provisionally was justified.

It was found, however, that if air at atmospheric pressure was admitted to one compartment the absorption was decreased by more than one half. This is just the opposite of what I found three years ago, when I discovered that I could photograph the 2536 absorption line by passing the light of a cadmium spark through a tube 3 metres long containing mercury vapour at room temperature, if the tube contained air at atmospheric pressure, while no trace of the line appeared if the tube was exhausted. There is, however, no real discrepancy, for the effect of the air, as I have shown previously, is to broaden the absorption line. This circumstance wats discovered independently by Angström in the case of $\mathrm{CO}_{2}$. The present experiments appear to show as well that, while the line is broadened, the intensity of the absorption at the centre of the line is materially reduced. I have observed the sume thing with iodine vapour, the lines becoming furzy and less black when air is admitted to the tube.

The failure to obtain the absorption line in the earlier experiment with the three-metre tube exhausted was of course simply due to the insufficient resolving power of the spectrograph employed.

In the experiments with the double cell which I have just described, I was unable to find any distinct evidence that

Plil. Mag. S. 6. Vol. 23. No. 137. May 1912. 3 A 
the resonance radiation contributed to the darkening of the photographic plate, for there appeared to be no difference between the case of a plate placed close against the cell and one placed at a distance. There is reason to believe that the radiations emitted by a resonator lag in phase belind the phase of the primary wave by $180^{\circ}$, that is they should reduce the intensity of the primary wave by interference. This is a delicate matter to investigate experimentally, but $\mathrm{I}$ have made one or two attempts. If we place a black sereen perforated with two small holes in front of the cell, we can limit the radiation which traverses the cell to two beams of small cross-section, each compartment transmitting one of them. The primary wave will now record itself on the plate as two small spots of the same diameter as that of the apertures. The short column of vapour traversed by the narrow beam in the high vacuum compartment gives off scattered resonance radiation, and we should expect this to record itself as a faint penumbra around the sharply defined image imprinted by the primary beam. No trace of any such penumbra was found, from which we can infer that the effect is negligibly small in comparison with that of the primary wave. This made me suspect that, even when the vapour is in a high vacuum, some true absorption of energy must take place, for it is obvious that the magnitude of the effect due to the resonance radiation will decrease in proportion to that of the primary wave as the factor of true absorption is introduced. It appeared possible to determine in this way, just what proportion of the energy diverted from the primary beam was absorbed and what proportion was scattered. I accordingly placed the quartz lens at the centre of the box, with the plate and absorption cell at the end opposite to that on which the porforated paper cone was fastened. This gave me a sharply focussed image of the circular aperture in the cone on the photographic plate, which was placed as before with the film in contact with the anterior quartz window. I now got a very intense beam of light from the resonance lamp, and a very black spot upon the plate with a distinct trace of a corona around it due to the seattered ratiation (Pl. XII. fig. 10). Air was now admitted to the cell and the experiment repeated. No trace of the corona appeared as was to be expected, as is shown in Pl. XII. fig. 11 .

This experiment will be better understood by reference to Pl. X. fig. 5, in which the scattered rays which produce the corona are represented by arrows. The question now is, how great an effect from these scattered rays are we to expect in comparison with the effect produced by the primary beam 
after its passage through the cell? A rigorous calculation involves some difficulties, but we can make an approximate estimate in the following way. In the experiment as it was tried, the thickness of the cell was $10 \mathrm{~mm}$., and the intensity of the primary beam was therefore reduced to one quarter of its original value, or in other words 75 per cent. of the energy was seattered, and 25 per cent. reached the plate as direct rays and recorded itself on a circular area $4 \mathrm{~mm}$. in diameter. It is evident that the intensity of the scattered resonance radiation from the first layer of molecules encountered by the primary beam will be most intense, but its intensity will be reduced when it reaches the plate, by its passage through the gas, in the same proportion as is the intensity of the primary ray, which excites the last layer of molecules to a much feebler luminosity. The intensity of all of the layers as seen by the photographic plate would thus appear to be equal. It is now evident that the 75 per cent. of the energy diverted from the primary beam, since it is given out in all directions, is distributed over a complete sphere, and we shall probably go not very far wrong if we assume it all as coming from the first layer, which will make our sphere of radius equal to $10 \mathrm{~mm}$. (the thickness of the cell), for in the actual experiment the photographic plate was placed in contact with the anterior window, and not at a distance as shown in the figure. The area of the sphere is $1256 \mathrm{~mm}$. and the area illuminated by the primary beam $1.2 \mathrm{~mm}$., the ratio of the areas being as 1 is to 105 . We have then a radiation of intensity 3 distributed over area 105 (scattered), and radiation of intensity 1 concentrated on area 1 . 'The direct image ought, therefore, to be about 35 times as bright as the corona. I made a rough measurement of this ratio and found it to be in the neighbourhood of $1: 40$, which agrees as well as we could expect, considering the way in which the calculation was made. The calculation was made on the assumption that no true absorption occurred, and the fact that there is some agreement between the calculated and the observed ratio indicates that we are dealing with pure scattering when the mercury vapour is in a high vacuum. The destruction of the corona by the admission of air is the direct consequence of the introduction of the factor of true absorption. I am of the opinion, however, that the ratio of the intensity of the secondary radiation to that of the primary, furnishes us with a better method of determining the ratio, and as I have already mentioned, the value obtained leads me to think that in a ligh vacuum there is no true absorption. A rigorous calculation of the ratio under specified conditions $3 \mathrm{~A} 2$ 
is much to be desired, and could be easily tested by experiment. The calculation could be made for a beam of either rectangular or circular cross-section. I believe that a beain with a square cross-section will prove best, and it must be remembered that in the experiment we photograph the entire mass of the gas, as seen from the side. I found that with a beam of this description the intensity of the secondary radiation elose to the beam of primary radiation was in some cases equal to one quarter of the value of the latter, though it fell off very rapidly in value as the distance from the primary beam was increased.

It thus appears that the destruction of the secondary resonance radiation is the result of the introduction of the factor of absorption, but it seemed best to test other hypotheses if possible. The only alternative that I have been able to think of is the possibility that the presence of the air alters the wave-length of the emitted light slightly so that it is no longer capable of exciting the vapour. The change necessary to accomplish this would be very slight, too small in all probability to be detected by any spectroscope except those of the highest resolving power. Another method, equally satisfactory and involving less difficulty than the use of a spectroscope, occurred to me. This we may call the method of the differential vacuum. A double cell with a quartz partition was constructed, of the form shown in Pl. X. fig. 6. Each compartment contained a drop of mercury, and the monochromatis light from the arc was focussed at the centre of the lower compartment, which was exhansted to a pressure of $6 \mathrm{~mm}$., previous experiments having shown that the secondary radiation is practically unnoticeable at this pressure. The upper compartment was highly exhausted. The point at which the rays were focussed was brought as close as possible to the under side of the horizontal partition separating the two cells, and as close as possible to the window through which the radiation was photographed, to avoid the loss of light due to an intervening layer of vapour. If now there is a change of wave-length due to the air in the lower compartment, the light (emitted by the directly excited vapour) which passes up through the quartz partition will be unable to stimulate the vapour in the upper compartment. If, on the contrary, the absorption hypothesis is correct, the vapour in the upper compartment will glow, since we have eliminated absorption here by the complete removal of the air. The photograph showed the secondary radiation in the upper compartment, and the ratio of its intensity to that of the primary was about the same as 
when both compartments were completely exhausted. This disposes of the second hypothesis, and is fully in accord with the absorption explanation.

\section{Transilion from Irregular Scattering to Regulur Reftexion by the Molecular Resonators.}

I have already alluded to the selective reflexion of monochromatic light by very dense mercury vapour, which I made a study of some years ago, and the transition from diffuse scattering to this regular reflexion has been made the subject of experimental investigation. Regular reflexion can occur only when the resonators are so closely packed together that the primary wave is stopped completely by a layer the thickness of which is of the order of magnitude of the wavelength. If the resonators are so sparingly distributed in space that the incident wave penetrates to any appreciable depth, their radiations do not combine to form a reflected wave in the usual sense of the term. Planck, I think, has somewhere mentioned the circumstance that the radiations from the resonators destroy the primary wave on account of their lag in phase, but that those in the opposite direction, there being no primary wave travelling in this direction with which they interfere, give rise to a wave which constitutes selective reflexion. If this occurred, except in the special case in which the wave is stopped at the boundary, it would constitute what we might term "volume reflexion" as contrasted to surface reflexion, but the phenomenon has no existence, I feel sure. It seemed so easy, however, to get evidence of volume reflexion if it existed, that I made one experiment, rather with the idea of convincing myself of its non-existence than anything else. A cell was made of the form shown in Pl. X. fig. 7 and a divergent beam passed into it through the oblique quartz plate. If even a very small percentage of the energy emitted by the resonators was returned towards the source, i.e., in the direction of the primary rays reversed, we should bave a converging system of rays which would be in part reflected from the oblique quartz plate and come to a focus on the photographic plate P. The plate, however, showed only a uniform darkening due to the light irregularly scattered by the resonators.

We do, however, obtain regular reflexion when the density of the vapour is so great that the wave can penetrate only to a very small depth, and I next investigated the subject of the density at which the change from scattering to regular reflexion takes place. A quartz bulb containing 


\section{Prot. R. W. Wood on Selective Reflexion, Scattering}

a drop of mercury, exhausted and sealed, was mounted just above a vertical chimney of sheet iron which served to immerse the bulb in the hot gases rising from a large Bunsen burner. A high temperature quartz thermometer was mounted with its bulb in contact with the quartz bulb, and by regulating the height of the flame of the burner, the bulb could be kept at any desired temperature. A divergent heam of the 2536 light was thrown upon the bulb by the quartz spectrograph, and the bulb photographed with the quartz camera. At room temperature the entire interior of the bulb was shown by the photograph to be filled with a uniform glow. As the temperature rose this glow was confined to the wall which received the direct rays from the spectrograph, owing to the failure of the radiation to penetrate to the interior. As the density of the vapour increased this glow appeared to draw in or close around the bright point which represented the image of the source reflected from the inner wall of the bulb. 'T'wo of these points usually appear in the picture, one reflected from the outer, the other from the inner wall. They are the virtual images of the source in the spherical mirror formed by the wall of the bulb. This contraction of the glow struck me as very peculiar, as it reminded one of the reflexion from a slightly corrugated surface, or a surface which has heen very finely ground but not polished. I obtained it with the bulb and with a small flask. PI. XI. figs. 5 and 6 show the phenomenon, the pressure of the mercury vapour being recorded on each photograph. I made a calculation by elementary methods of what was to be expected when the resonators became more closely crowded together, and came to the conclusion that such a drawing-in or contraction of the glow was not to be expecterl, but that what would actually occur would be a gradual diminution of the intensity of the glow, which at low pressures is spread uniformly over the illuminated wall, and a simultaneous increase in the intensity of the small image reflected from the inner wall. I now prepared a new bulb, which had never been heated or used in any experimental work, and found no trace of the contraction of the glow. A careful examination of the old bulb and the flask in a strong light revealed the presence of a slight amount of devitrification of the inner surface, the reflected image of the sun appearing surrounded with a fairly bright halo. This circumstance at once explained the contraction of the glow with increasing vapour pressure. As the density of the mercury vapour increased, the reflecting power of the inner wall increased, much as if a deposit 
of a metal was being gradually thrown down upon it, and this increase of the reflecting power was sufficient to develop the diffraction halo due to the slight roughness of the inner wall. The fact that the contracting glow usually had a definite though irregular shape, and was frequently accompanied by neighbouring patches of lesser intensity, mate me suspicious of the phenomenon in the beginning, but it was not until $\Upsilon$ noticed the slight devitrification of the surface that I was able to give a satisfactory explanation of the thing. In Yl. XI. fig. 6, the last impression was taken after the mercury arc had been burning for half a minute, and no longer emitted the exact frequency for which the mercury molecules responded. The reflexion in this case is due wholly to the quartz, and we see only the two minute points of light previously referred to.

'The real phenomenon is shown in Pl. XI. fig. 7, made with the clean bulb. A and B were taken at room temperiture, the former with a five-second exposure, the latter with one of half a second, to bring out better the relative intensities of the two images reflected from the inner and outer walls. I threw these out of focus a little, so that their relative intensities could be better determined. 'They appear expanded into circles of light due to zonal errors of the lens. It is clear that at room temperature the lower circle is much brighter than the upper, which was the one reflected from the inner wall. The other pair of circles are reflected from the back wall, and disappear in the other pictures owing to the failure of the radiation to penetrate to the back. Pictures $\mathrm{C}$ and $\mathrm{D}$ were made with a pressure of mercury vapour of about $20 \mathrm{~mm}$. The glow is now confined to that portion of the wall which receives the direct rays, and the image formed by the outer wall is still brighter than that formed by the inner, the ratio being about the same. $\mathrm{E}$ and $F$ were made with a pressure of very nearly one atmosphere. The diffuse glow has entirely disappeared, and there remain only the two regularly reflected images, the upper circle now being distinctly brighter than the lower. That this image (the upper circle) really came from the inner wall was proved in the following way. The heated bulb was cooled on the illuminated side by a blast of air. This caused a very fine "dew" of mercury globules to condense on the wall, practically silvering it on the inside. A photograph was immediately taken and the upper circle of light was found to be the brighter of the two, as was the case with the dense mercury vapour. The diffuse reflexion begins to 


\section{Prof. R.W.Wood on Selective Reflexion, Scattering}

weaken at a pressure of about $2 \mathrm{~cm}$. and is practically gone at $70 \mathrm{~cm}$.

\section{Polarization Experiments.}

Inasmuch as I have discovered strong polarization in the fluorescent light emitted by sodium, potassium, and iodine vapour, I fully expected to find it in the case of the mercury resonance radiation, as the mechanism of the emission would appear to be much simpler in this case than in the other cases where we bave, in addition to the resonance radiation, other associated frequencies emitted, which give rise to what I have named resonance spectra. No trace of any polarization could be detected with a Babinet compensator mounted in front of the resonance lamp. A small Foucault prism was used as an analyser, as ordinary Nicol's prisms are opaque to the ultra-violet on account of the Canada balsam with which they are cemented.

\section{Experiments with the Resonance Lamp.}

The radiation emitted from the exhausted quartz bulb is so homogeneous, that a layer of mercury vapour $5 \mathrm{~mm}$. thick and at the pressure which it has at room temperature $(0.001 \mathrm{~mm}$.) reduces its intensity by about one hall. Various investigations with the vapour at exceedingly low pressure at once became possible. It is as if we had a gas which appeared quite black even at pressures commonly employed in vacuum-tubes. It will be possible to study the rate at which the vapour diffuses into other gases at low pressures, and it may be possible to tell in this way whether the resonators are in reality mercury molecules or larger aggregates.

I made two photographs which illustrate what a sensitive detector of small traces of mercury vapour we have in the light of the resonance lamp. A quartz bulb having an internal diameter of $1.5 \mathrm{~cm}$., containing a drop of mercury, was mounted in front of a photographic plate in a dark box and illuminated with the light of the lamp. The bulb cast a shadow as black as ink. The bulb was now opened, the mercury drop removed, and the bulb washed out with nitric acid and distilled water, heated nearly red-hot and a blast of air blown into it, and again photographed. It still gave the black shadow, though not as black as before. It was only by prolonged heating to a red-heat and much rinsing out with an air current that I was able to obtain a shadow picture which showed the flask transparent. The flask had been 
previonsly heated until the mercury rapour in it had at fressure of several atmospheres, and I imagine that the inner surface may have adsorbed some of the vapour, which was not removed by the acid. A chemist would undoubtedly have called the flask clean, after the first treatment which I gave it. The two photographs are reproduced on Pl. XII. tig. 8 .

I next drilled a shallow cavity in the end of a brass cylinder, warmed it to a temperature of perhaps ten degrees above the temperature of the room, and placed a drop of mercury in the cavity, the drop standing up above the level of the end of the cylinder. This was photographed in the dark box by the light of the lamp, and the picture showed the black column of mereury vapour carried up by the convection current of warm air (PI. XII. fig. 9).

$\mathrm{I}$ am at present setting up a Michelson interferometer with a fluorite plate coated with a cathode deposit of gold for the purpose of ascertaining how large a difference of path is possible in the case of interference fringes formed by the light of this lamp. I imagine that the light may turn ont to be more homogeneous than that of any other source with which we are acquainted. Of course it is possible that the line itself is complex, in which case matters will be different. The dispersion of the vapour in the vicinity of the 2536 line will also be investigated with the same apparatus. It will be interesting also to place the resonance lamp in a powerful magnetic field, and study the Zeeman effect for a gas radiating by resonance instead of under the action of an electrical or other stimulus.

It even appeurs possible that the electric analogy of the Zeeman effect can be detected with the resonating mercury vapour, since it can be brought to a high luminosity in a vacuum which is practically non-conducting, that is if the rapour does not become conducting when excited hy the ultra-violet light, which I doubt; for a roughly carried out experiment with a small gold-leaf electroscope showed no conductivity when the vapour in the bulb between two electrodes was excited. We cannot infer from this what it would do with a potential sufficient to give say a $20 \mathrm{~cm}$. spark in an alternating spark-gap, but I have a feeling that the vacuum would support it.

In fact a very wide field of investigation appears to be opened by the discovery of the very remarkable behaviour of the vapour of mercury at very low pressures.

This paper forms the third of a series upon the optical 


\section{Profs. Wellisch and Bronson on the Distrilution of}

properties of mercury vapour, the first being upon the fluorescence, etc. of the vapour (this journal, vol, xviii. p. 240), and the second, on the selective reflexion of monochromatic light by mercury rapour (tom. cit. p. 187). The investigation has been made possible through a grant from the Rumford fund of the American Academy of Arts and Sciences, and my thanks are due to the members of the Rumtord committee for their generous aid.

LXVIII. The Distribution of the Active Deposit of Radium in an Eletric Field. By E. M. W ELLISOH, Assistunt Professor of Physics in Yale College, and H. L. Bronson, Professor of Physics in Dalhousie Colleye, Halijax* *

\section{Introductory.}

rTHE present paper contains the results of a series of experiments which were carried out at the Sloane Physical Laboratory of Yale College, and which were undertaken with a view of throwing light on the mechanism involved in the transmission of the active deposit of radium to the electrodes in an electric field or to the exposed solid surfaces in the absence of such field. In order to acconnt for this transport of activity one of us $\dagger$ had already suggested a theory in which the view was taken that the transmission was effected as a result of the interaction between the active deposit particles (or restatoms) and the ions formed in the gas by the radiation accompanying the radioactive disintegration; in particular, an attempt was made to explain on this theory the experinental result obtained by Rutherford $\ddagger$ and Franck $\S$ that the restatoms moved through the gas with the same velocity as the positive ions.

The original object proposed in the present experiment was to ascertain whether the distribution of activity on the electrodes could be affected in any way by the application of an extranecus source of ionization such, for instance, as that produced by causing intense Röntgen rays to pass through the gas.

We might be permitted to anticip ate here the results of the present experimental investigation and to state that, although the application of such extraneous source of ionization has so far been found to produce little or no effect on

* Communicated by the Autliors.

$\dagger$ Wellisch, Ierh. Deuts. Phys. Ges. xiii. p. 159 (1911).

* Kutherford, Fhit. Mar. ser, 6, r. p. 90 (1903).

\$ Franck. Terh. Deuts. Phys. Ge's. xi. p, sot (1000). 

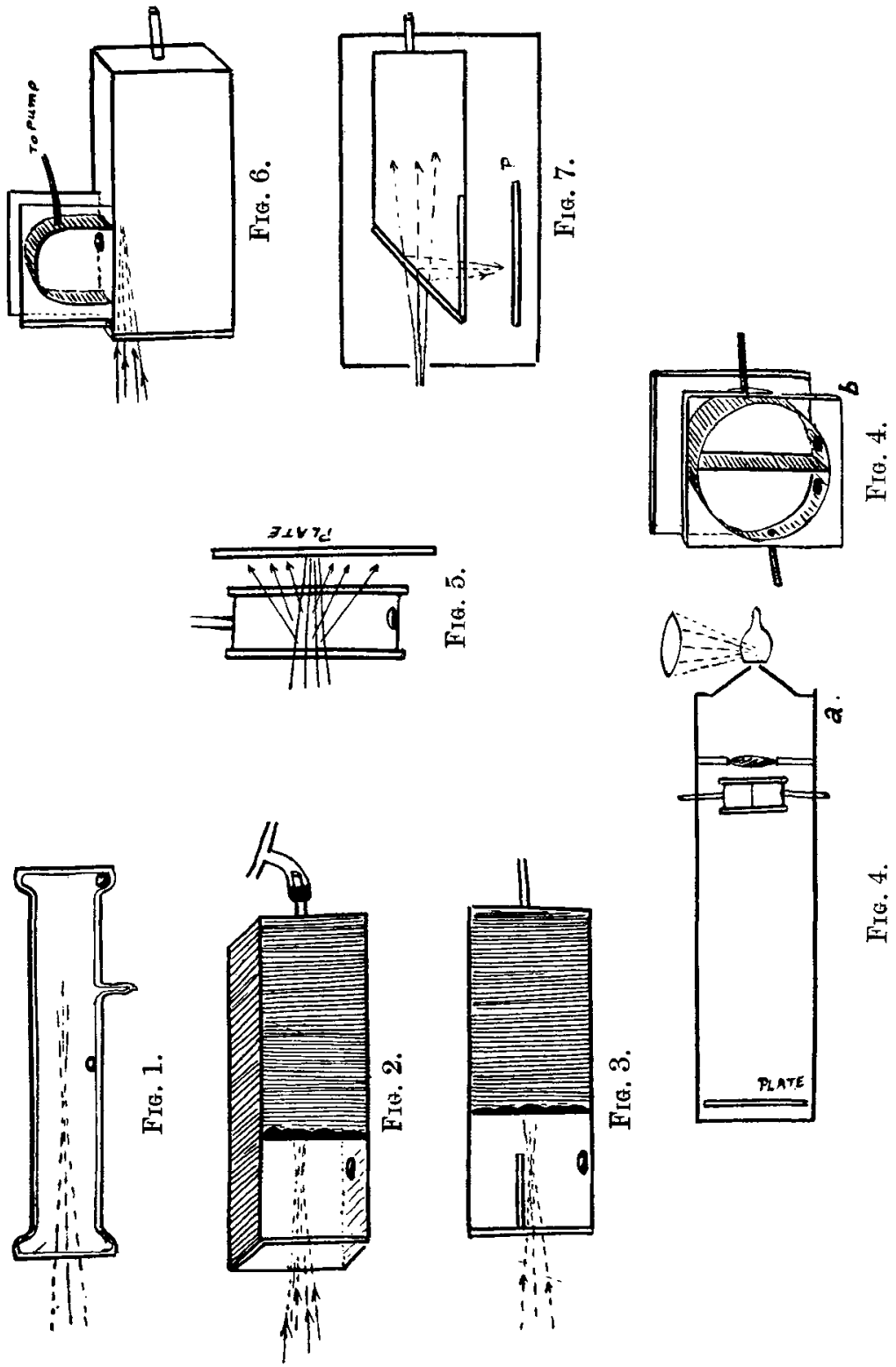


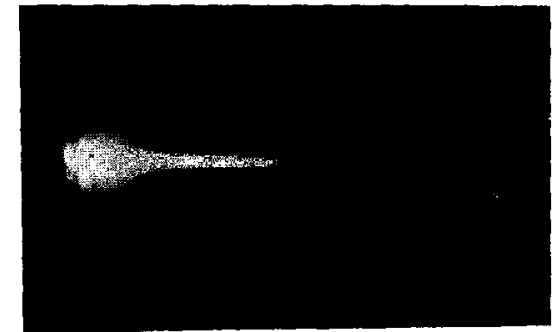

FIG. 1.

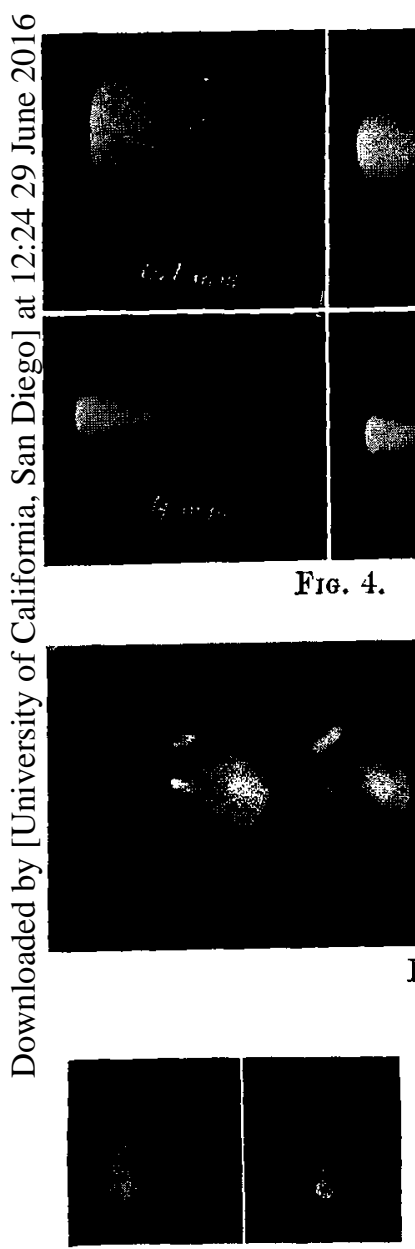

A

B

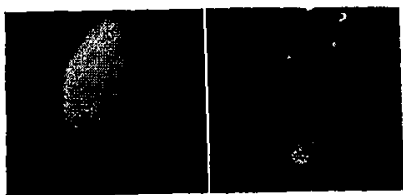

C

D

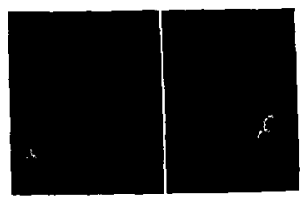

$F_{1(i ;} ;$

FIG. 2.
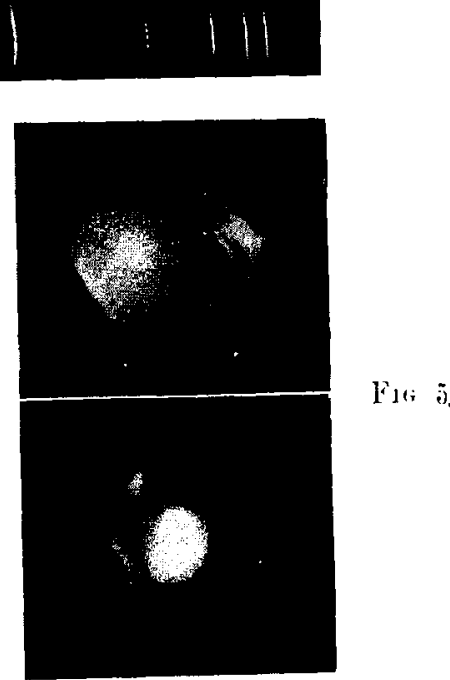

Fig. 6.

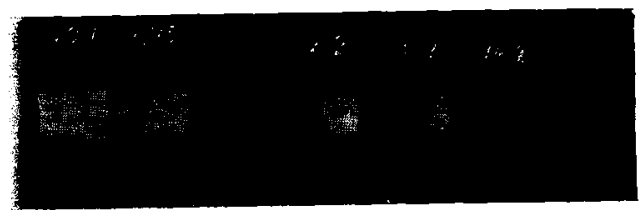

E

Frg. 3

$F_{I}: ., i$

FIG. 12. 

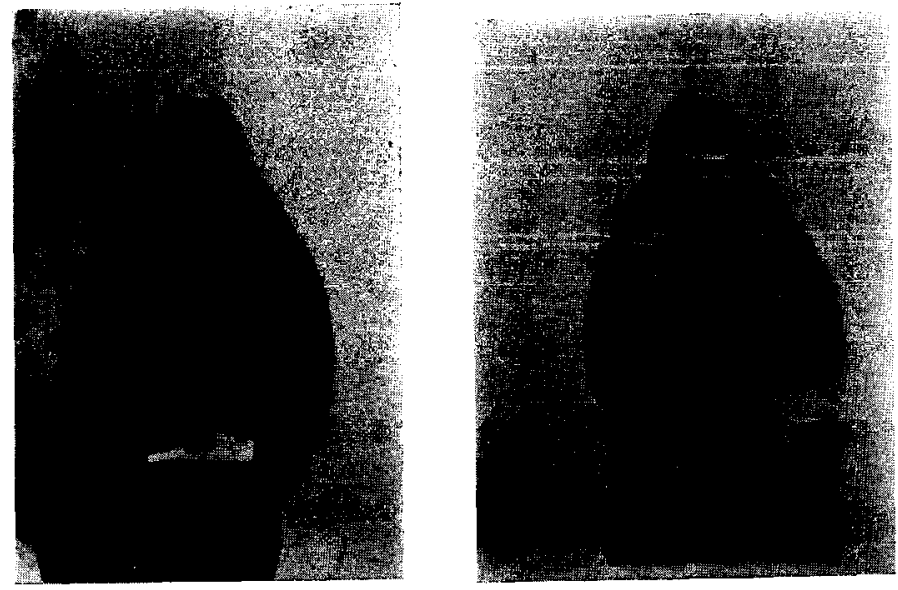
Fig. 8.

Fig. 11.

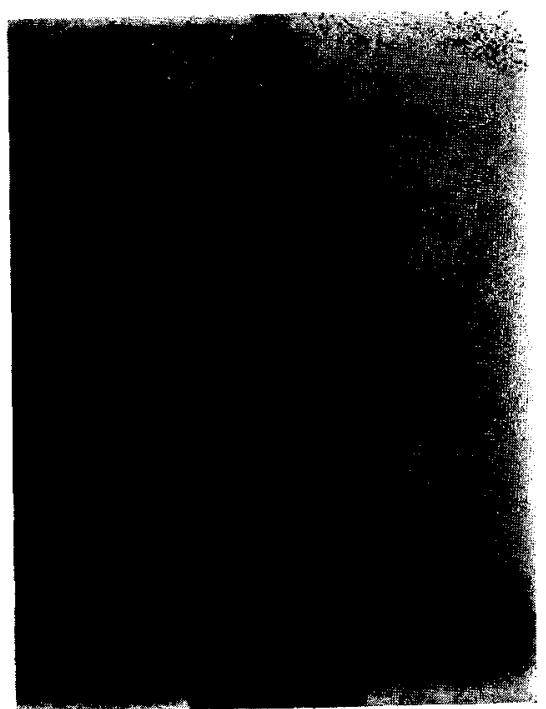

FIG. 9.

Fit. 10. 